\title{
Double-contrast method for the evaluation of gastric lesions
}

\author{
TORIOLA F. SOLANKE, ${ }^{1}$ KENJI KUMAKURA, MASAKAZU MARUYAMA, \\ AND NAIKI SOMEYA
}

From the Department of Internal Medicine, Cancer Institute Hospital, Tokyo, Japan

Despite extensive experience gained in the radiography of the abdomen the degree of accuracy in determining lesions of the stomach is far from ideal and has stimulated the development of new diagnostic modalities. Of these, the double-contrast method deserves some mention. In Japan, where this method is extensively practised, it has been possible to detect very early cases of carcinoma of the stomach (Shirakabe, 1966; Kurokawa, Kajitani, and Oota, 1966). This paper focuses attention on the importance of this technique by way of illustrative cases and describes a procedure which could be part of the routine radiological examination of the stomach.

\section{METHOD}

The technique described in this paper is that practised at our hospital. The patient is advised not to drink or have breakfast before examination which is usually done in the morning. Under fluoroscopic control the patient drinks a mouthful of barium sulphate suspension and a mucosal study is carried out in both the supine and prone positions. While in the upright position the patient takes 200 to $300 \mathrm{ml}$ of barium sulphate suspension and a barium-filled film is taken. The table is tilted to the horizontal position, and the stomach is insufflated with 200 to $300 \mathrm{ml}$ of air through a nasogastric tube. The tube is then removed. The amount of air put into the stomach is dependent on the quantity already present. The total amount of barium sulphate and air usually needed varies between 400 and $600 \mathrm{ml}$. After the insufflation the patient is immediately and quickly rolled from side to side to ensure that the mucosa is well coated with barium. This step is repeated as often as necessary throughout the examination.

The examination is then conducted in the following sequence: (1) supine, (2) right oblique, (3) left oblique, and (4) upright positions. These are the important positions although it may be necessary to place the patient in the head-down or foot-down position. Compression studies are carried out next. If an anterior wall lesion is suspected during the mucosal study, the prone

${ }^{1}$ Present address: Department of Surgery, The University of Ibadan, Ibadan, Nigeria. double-contrast method, as described by Kumakura, Fuchigami, and Takagi (1965), is done.

\section{ILLUSTRATIVE CASES}

CASE 1 (M.A., 85/65) A woman aged 61 was admitted to hospital with a history of loss of weight, epigastric fullness, and belching for 20 years. She gave a past history of two severe episodes of haematemesis. Physical examination on admission revealed epigastric tenderness; her blood pressure was $130 / 60 \mathrm{~mm} \mathrm{Hg}$.

Marked shortening of the lesser curvature (tea-pot deformity) was demonstrated in the barium-filled film (Fig. 1a), while the double-contrast films showed a linear niche in the region of the angle (Fig. 1b). Macroscopic examination of the operation specimen (Fig. 1c) revealed a long linear scar in the vicinity of the pylorus placed at right angles to the lessure curvature. There was an ulcer at the anterior wall end of the scar.

The appearance of a grossly deformed and shortened lesser curvature in a barium-filled film should lead to the suspicion of a linear scar, which is best demonstrated by the double-contrast technique. It is impossible to see the whole extent of the scar during endoscopy because of the deformity of the lesser curvature.

CASE 2 (K.I., 313/66) A 50-year-old woman was admitted to hospital with a history of epigastric pain on and off for five or six years. Four weeks before admission she had a severe haematemesis. Physical examination on admission revealed no abnormalities.

The barium-filled film showed a relatively large niche in the lesser curvature of the body (Fig. 2a). Doublecontrast films revealed the smooth margins of the niche, and the mucosal folds running to the edges of the niche (Fig. 2b). On endoscopic examination an ulcer was seen on the lesser curvature. Naked-eye examination of the resected specimen showed an ulcer crater on the lesser curvature of the body and the converging folds, mostly from the posterior wall (Fig. 2c). The ulcer crater was oval and measured $5 \times 8 \mathrm{~mm}$.

The smooth margin of the niche and the converging mucosal folds shown in double-contrast films 


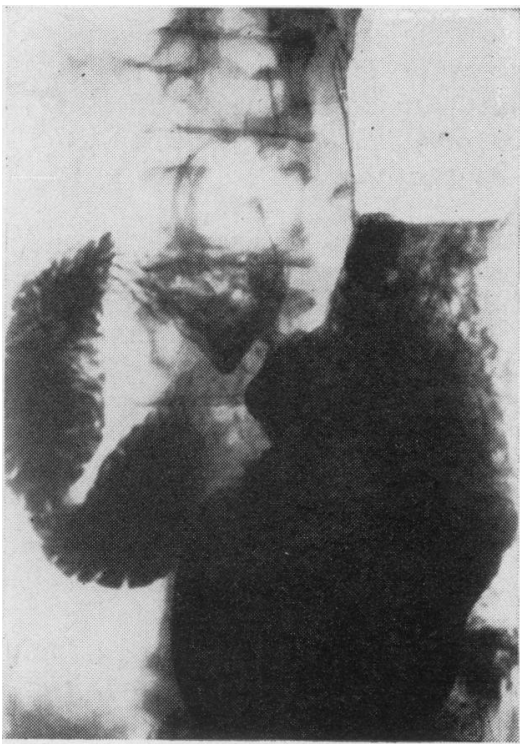

FIG. 1 a.

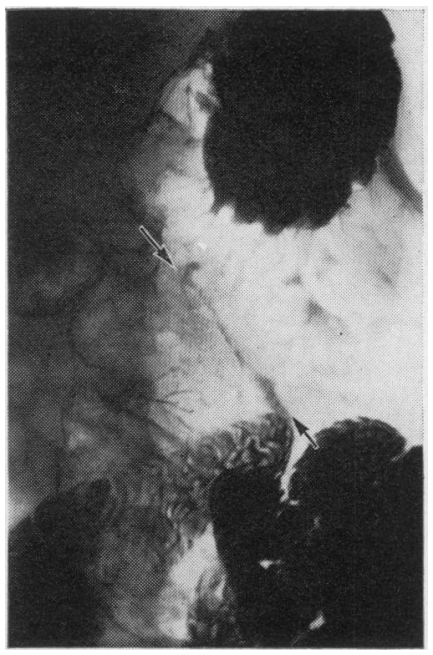

FIG. 1 b.

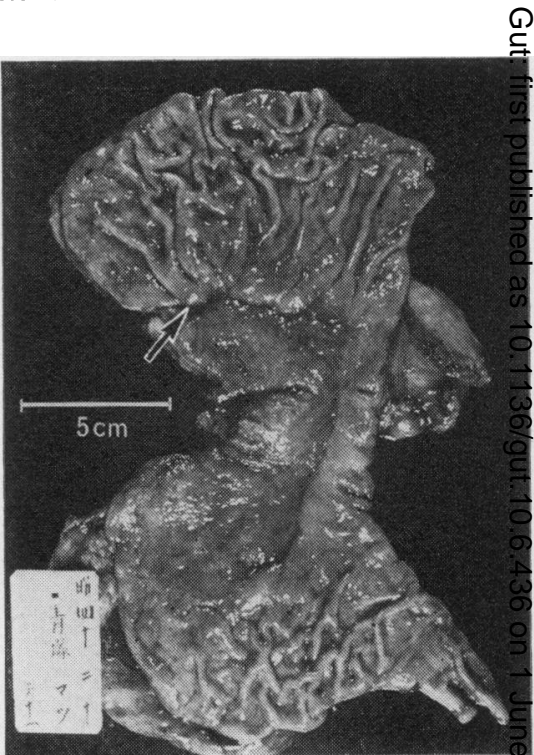

FIG. 1c.

FIG. 1a. Barium-filled film showing marked shortening and deformity of the lesser curvature.

FIG. 1b. A linear niche (arrowed) is shown in the double contrast film.

FIG. 1c. Resected specimen. The linear scar is placed at right angles to the lesser curvature. There is an ulcer (marked) at the anterior wall end of the scar.

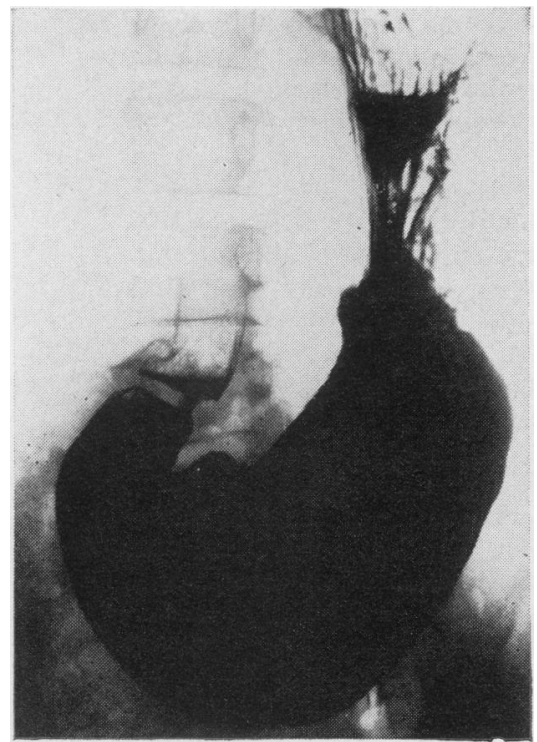

FIG. 2a.

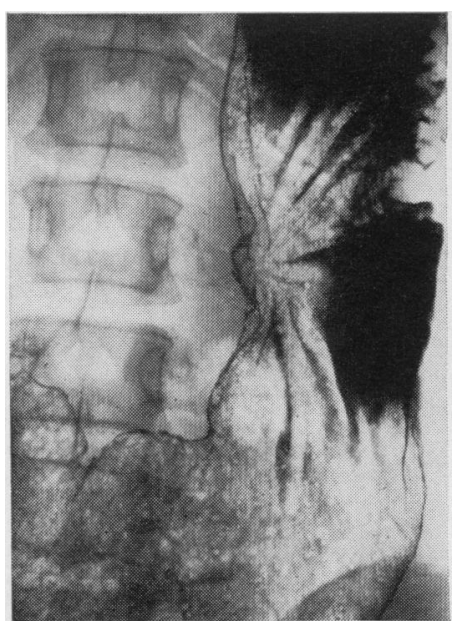

FIG. $2 b$.

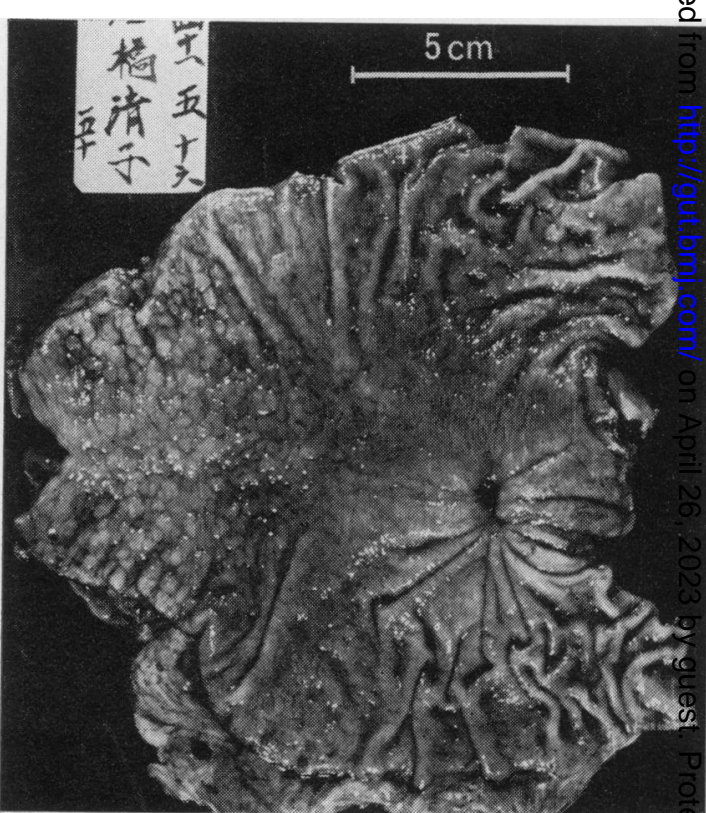

FIG. 2c.

FIG. 2a. Barium-filled film showing a large niche in the lesser curvature of the body.

FIG. 2b. Double-contrast film. Note the smooth margins of the niche and the mucosal folds running to the edges of the niche.

FIG. 2c. Operative specimen showing the ulcer crater. 


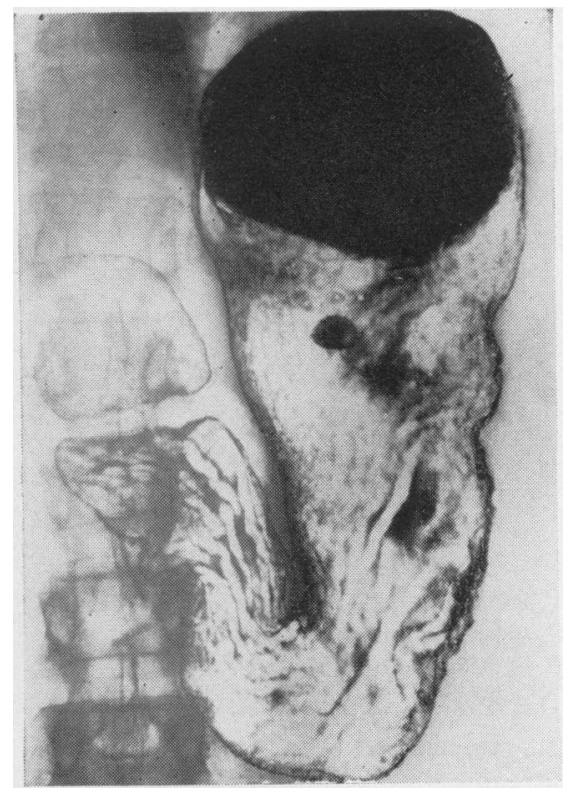

FIG. 3a. Doublecontrast film showing a mushroom-like shadow in the body of the stomach.

FIG. 3b. Prone double-contrast film showing the polyp.

FIG. $3 c$.

Resected specimen showing the polyp with $a$ large head and short stalk. should make one suspect the presence of a benign ulcer (Shirakabe et al, 1961). The contour of the niche and the shape of the mucosal folds due to a benign gastric ulcer can only be adequately demonstrated by the double-contrast method.

CASE 3 (T.S., 364/66) A 52-year-old woman was admitted to hospital with a two-year history of lower abdominal pain. Physical examination on admission revealed no abnormalities. Double-contrast films showed a mushroom-like shadow in the upper body of the stomach suggestive of a pedunculated polyp in the anterior wall (Fig. 3a). The stalk and head were clearly demonstrated by the prone double-contrast method (Fig. 3b). Naked-eye examination of the resected specimen revealed a polyp $(20 \times 8 \times 4 \mathrm{~mm})$ with a large head and a short stalk in the anterior wall of the gastric body (Fig. 3c). Histological examination showed an adenomatous polyp.

Polyps in the anterior wall of the stomach can be demonstrated by the supine double-contrast method. For better definition of the shape, head, and stalk the prone double-contrast method and compression study are necessary.

CASE 4 (B.K., 176/68) This 73-year-old man was found to have a gastric polyp on a mass screening survey. Double-contrast films showed a tumour in the body of the stomach (Fig. 4a) with irregular margins and surface, and gastric carcinoma type $\mathrm{IIa}^{1}$ was suspected. Endoscopic diagnosis was gastric carcinoma type I or IIa. Biopsy of the lesion confirmed the diagnosis of the carcinoma of the stomach. A tumour mass on the lesser curvature of the body was seen on naked-eye examination of the operation specimen (Fig. 4b). Histological examination showed papillotubular adenocarcinoma limited to the mucosa with no metastases.

The double-contrast method clearly demonstrated the irregular appearance and shape of the tumour. These features cannot be seen in barium-filled films unless compression studies are done.

CASE 5 (s.o., 743/67) This 41-year-old man was referred to the hospital from the mass screening survey clinic with a two months' history of loss of weight and epigastric fullness. Double-contrast films showed a shadow in the body of the stomach with an irregular margin, faintly filled with barium sulphate (Figs. 5a and b). There were irregular criss-cross markings in the floor of this area, with abrupt interruptions of the mucosal folds converging on the area. These appearances were suggestive of early gastric cancer, type IIc. This diagnosis was supported by endoscopy. The operation specimen (Figs. 5c and d) revealed, on macroscopic examination, a depressed area $(25 \times 45 \mathrm{~mm})$ in the posterior wall of the lower gastric body. The floor was nodular and irregular. Abrupt interruptions of the converging

${ }^{1}$ Classification of early gastric carcinoma by the Gastroenterological Endoscopy Society 1962. 


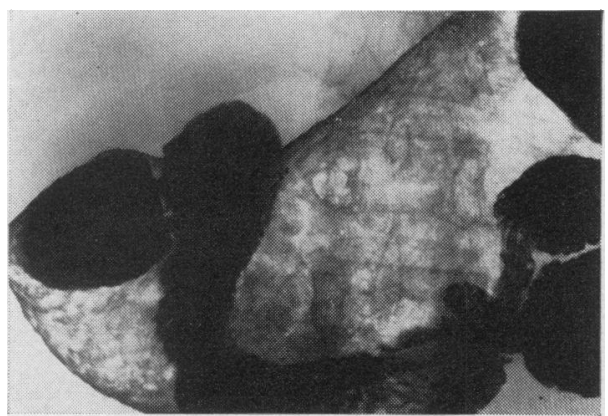

FIG. 4a.

FIG. 4a. Double-contrast film showing an irregular tumour in the body of the stomach. FIG. 4b. The resected specimen. The tumour is on the lesser curvature of the stomach.

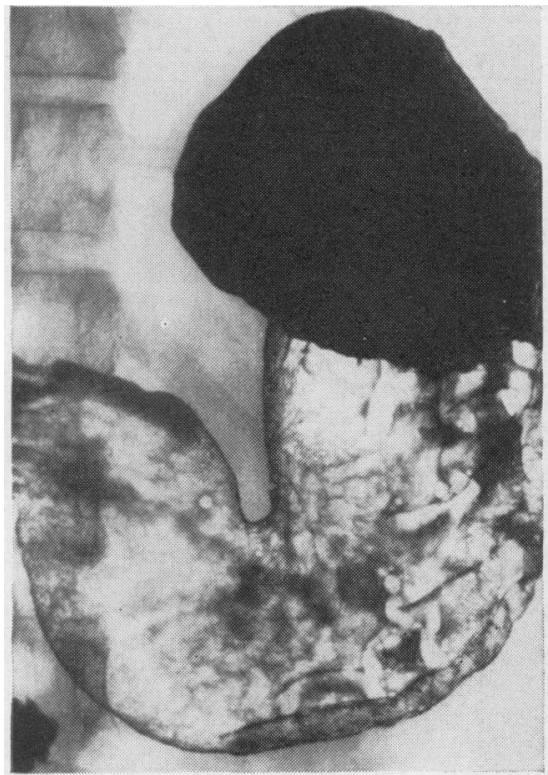

FIG. 5a.

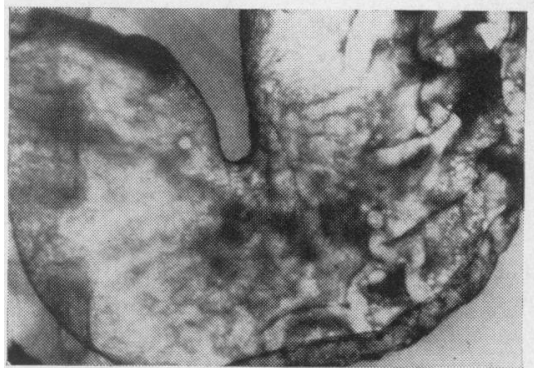

FIG. $5 b$.

FIG. 5a and FIG. 5b. Double-contrast films. Note the irregular area with criss-cross markings in the body of the stomach.

FIG. 5c. Resected specimen. Note the irregular area in the posterior wall of the gastric body and the abrupt interruption of the mucosal folds.

FIG. 5d. Higher magnification of Figure 5c.

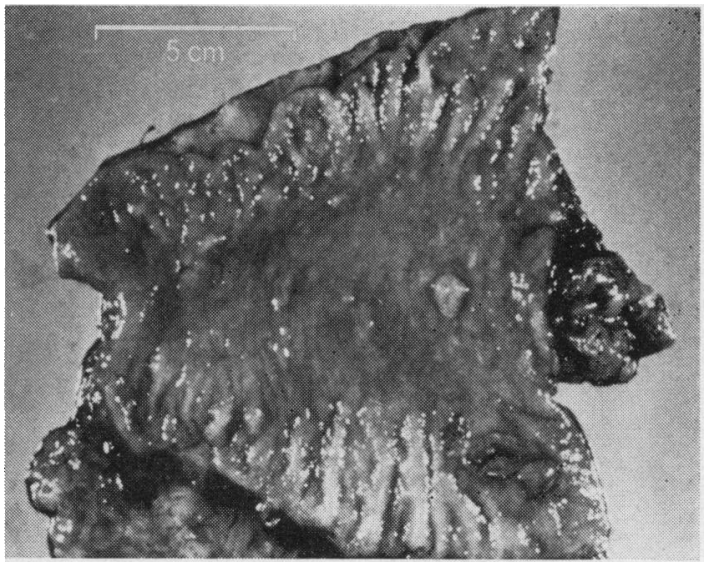

FIG. $4 b$.

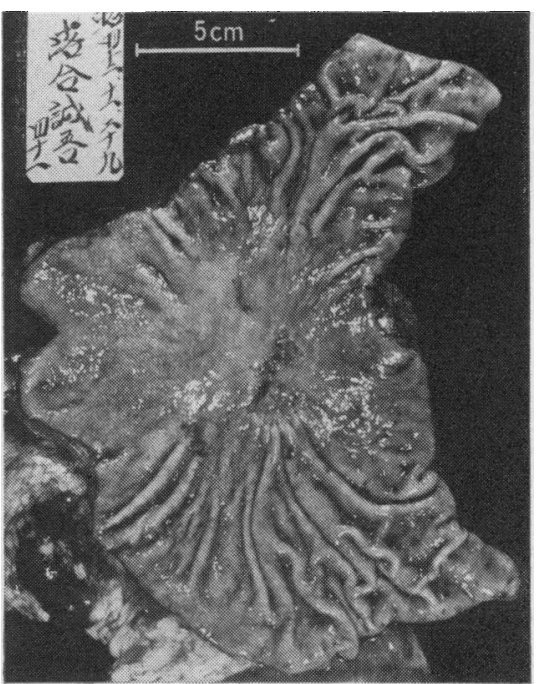

FIG. 5c.

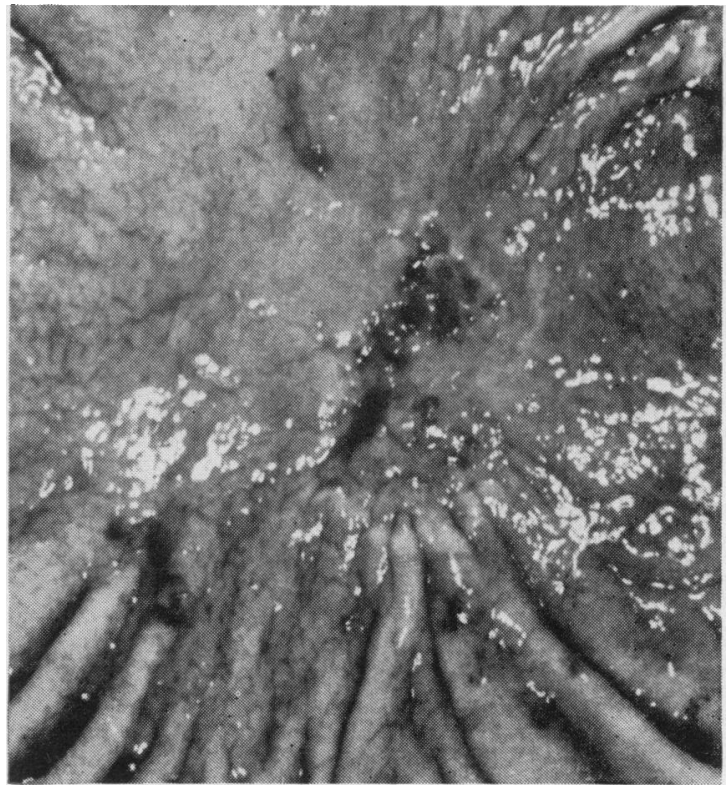




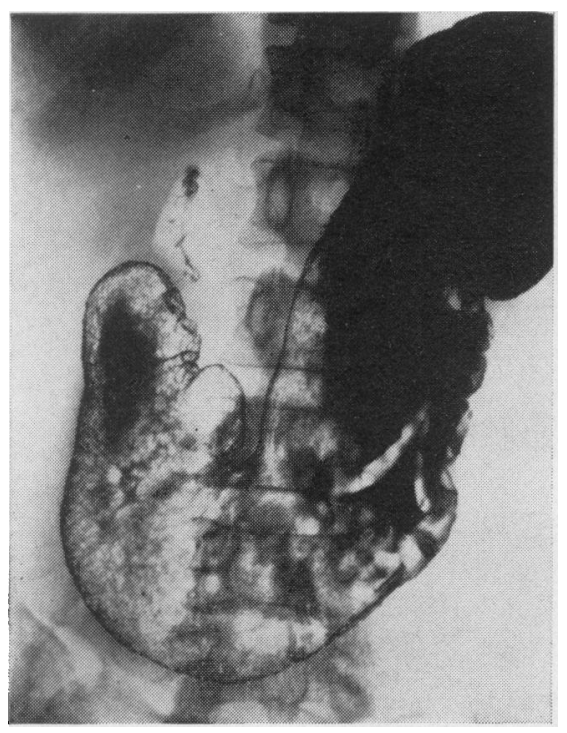

FIG. 6a.
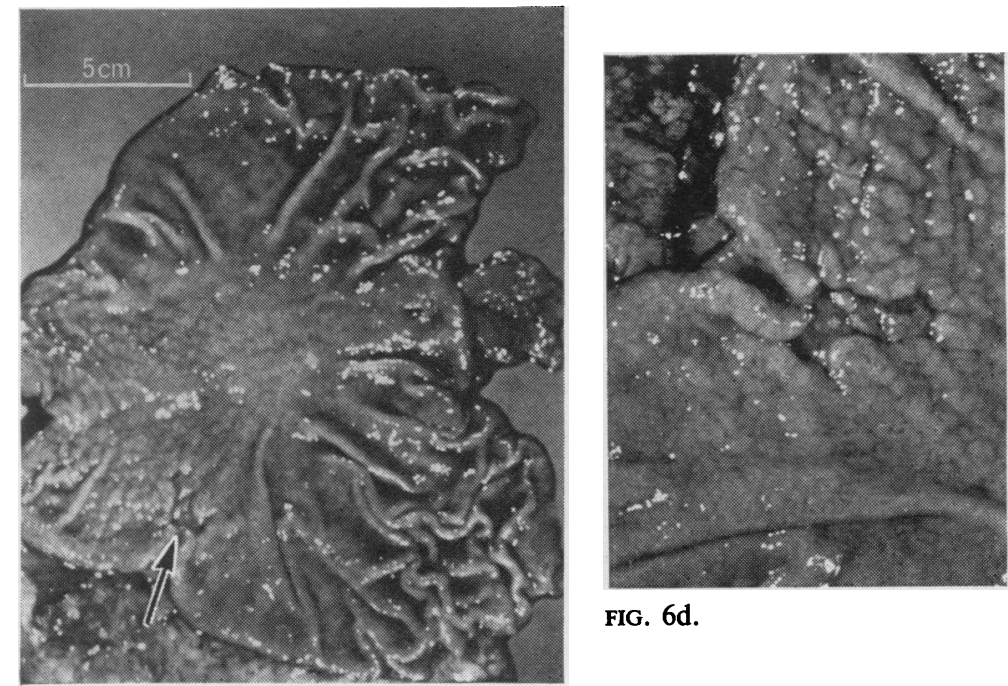

FIG. 6d.
FIG. 6a. Doublecontrast film showing a depressed shadow filled with barium in the antrum.

FIG. 6b. Higher magnification of Figure 6a.
FIG, 6c.

mucosal folds were noted. Histological diagnosis was scirrhous carcinoma type IIc + III.

The radiological features of type IIc early gastric cancer shown by this method may not be observed by the conventional methods.

CASE 6 (M.Y., 794/67) A 46-year-old woman complained of belching, a feeling of heaviness in the epigastrium, anorexia, and loss of weight for seven months. The double-contrast films showed a depressed shadow filled with barium in the antrum. The margins of this area were irregular with translucent areas in the centre (Figs. 6a and b). Type IIc gastric carcinoma was seen in the posterior wall of the antrum on endoscopy. Biopsy of the lesion through a fibrescope showed scirrhous adenocarcinoma. The resected specimen (Figs. 6c and d) showed, on macroscopic examination, an L-shaped, depressed area, $25 \times 14 \mathrm{~mm}$, in the posterior wall of the antrum. The floor was granular and the edges were irregular. The surrounding mucosa was slightly raised.
FIG. 6c. Resected specimen. depressed area in the antrum arrowed).

FIG. 6d. Higher magnification of Figure $6 c$. 


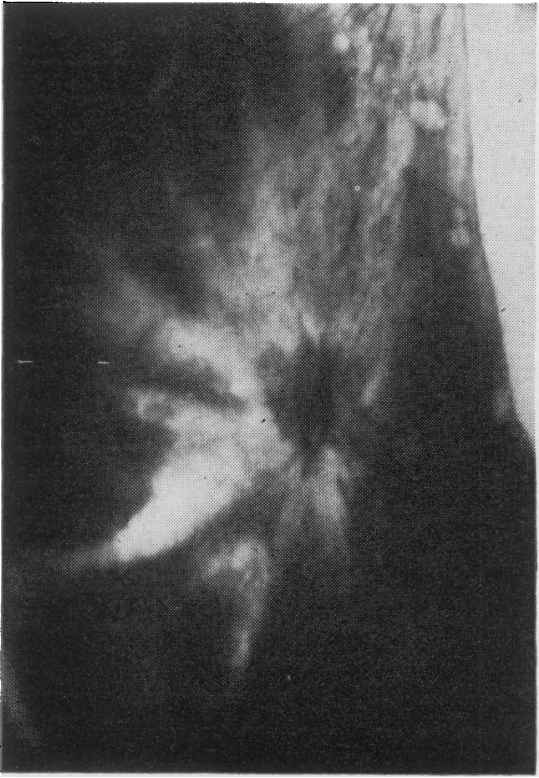

FIG. $7 \mathrm{a}$.

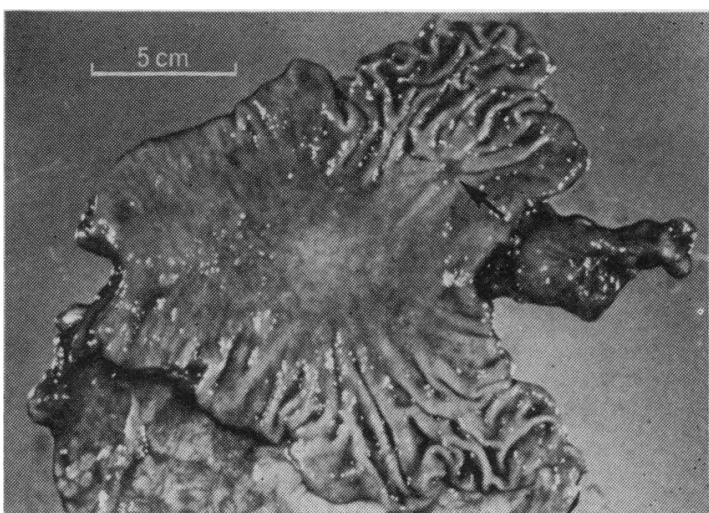

FIG. $7 b$.

FIG. 7a. Prone double-contrast film showirg an irregular depressed area in the anterior wall of the stomach. Note the abrupt endings of the mucosal folds around the depressed area.

FIG. 7b. Resected specimen. The mucosal folds ended some distance from the depressed area (arrowad).

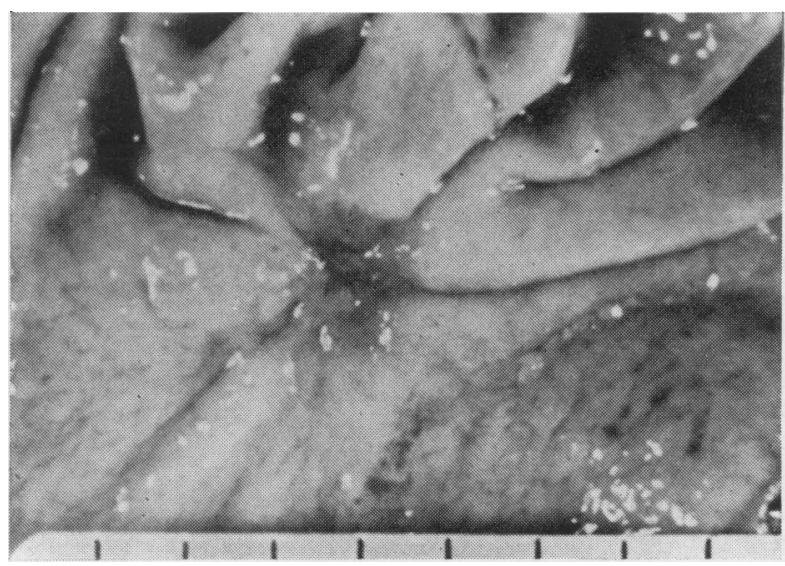

FIG. 7c. Higher magnification of Figure $7 b$.

Microscopic examination showed adenocarcinoma, mucocellular type IIc + III.

CASE 7 (K.F., 802/64) A 43-year-old man was admitted to hospital with four years' history of epigastric pain and discomfort. Prone double-contrast films (Fig. 7a) showed an irregular depressed area in the anterior wall of the body with surrounding translucency and converging mucosal folds. Type IIc gastric carcinoma was seen on endoscopy. Naked-eye examination of the operation specimen (Figs. $7 \mathrm{~b}$ and c) revealed a depressed area, $10 \times 10 \mathrm{~mm}$, with central ulceration in the anterior wall of the body. The converging mucosal folds ended some distance from the margin of the depressed area. Adeno- carcinoma (tubulo-medullare type) was demonstrated on histological examination.

A lesion of the anterior wall of the stomach can only be effectively demonstrated by the prone double-contrast technique and compression studies.

\section{DISCUSSION}

This double-contrast method of radiological examination of the stomach can be performed in a relatively short time with minimal discomfort to the 
patient. It gives better definition of the lesser and greater curvatures, and it is particularly valuable for the recognition of small lesions and early gastric carcinoma (Shirakabe, 1966; Kumakura et al, 1968), which might be missed by the conventional methods. It is possible to study the shape and extent of lesions in the stomach with some degree of accuracy. Multiple lesions are readily demonstrated by this technique.

The patient requires no elaborate preliminary preparation except for the restriction of drink and breakfast. Some workers recommend the administration of Buscopan (hyoscine N-butylbromide) intramuscularly 10 to $15 \mathrm{~min}$ before examination because it slows down the peristaltic movements of the stomach thereby allowing for better examination. Injection of Buscopan, on the other hand, may obscure the deformity of the stomach caused by linear and multiple ulcers. Its use is only recommended when detailed examination of the stomach is contemplated.

The important aspects in this technique are the administration of relatively large quantities of air and barium sulphate, and frequent changes of the patient's position. The amount of air needed must be assessed accurately during fluoroscopy. Overinsufflation makes recognition of small lesions difficult. Details of various parts of the stomach can be obtained by altering the patient's position. The upper body, fornix, and cardia can be studied effectively with the patient in the upright position. The right oblique position allows for thorough examination of the antrum, while the left oblique position, of the upper body and fornix. The supine position gives details of the lower and mid-body of the stomach.

Although advanced cancer, a large ulcer, and polyps in the anterior wall of the stomach may be diagnosed by the conventional methods, diagnostic difficulties arise in finding small lesions such as the superficial depressed type of early gastric carcinoma. For excellent detail of anterior wall lesions the prone double-contrast method is indispensable (Kumakura et al, 1968) and should be performed after the routine supine double-contrast examination has been completed.

The introduction of air into the stomach by the nasogastric tube is recommended, because with it the amount of air and barium in the stomach can be controlled. Excessive gastric juice, which tends to obscure small lesions and prevents mucosal coating with barium, can also be aspirated. Some authors (Taylor, Kevin, Macken, Bachman, and Seaman,
1965) advocate the use of air-producing tablets, but with these it is difficult to control the amount of air in the stomach. Moreover it takes some time for air to be released in the stomach and this lengthens the period of examination. Occasionally air is not released.

Compression studies may help in locating raised lesions in the antrum and mid-body, but not depressed lesions such as type IIc carcinoma of the stomach. The double-contrast method gives valuable information during examination of the upper body and fornix, where compression studies cannot be carried out. Review of double-contrast films when the examination is complete yields more information than that seen during fluoroscopy or felt during compression studies. For example, type IIc gastric carcinoma $30 \mathrm{~mm}$ or less in diameter is not usually visible during fluoroscopy, but can only be seen on examination of the double-contrast films.

Radiodiagnosis is an important and indispensable tool in the detection of early carcinoma of the stomach. By the double-contrast method gastric cancer, 4 to $5 \mathrm{~mm}$ in diameter, has been diagnosed preoperatively (Ichikawa, 1968).

\section{SUMMARY}

A double-contrast method for radiological examination of the stomach is described. Seven cases in which this method has been helpful in diagnosis are reviewed. The importance of this method is underlined.

We are grateful to Dr Kurokawa, Director of the Cancer Institute Hospital, Tokyo, for his cooperation in the preparation of this paper, and Mr Yamada of the medical illustration unit for the photographs.

\section{REFERENCES}

Ichikawa, H. (1968). Personal communication.

Kumakura, K., Fuchigami, A., and Takagi, K. (1965). X-ray diagnosis of diseases in the anterior wall of the stomach. Rinsho Hosha, 10, 787-796. (In Japanese.)

_-, Maruyama, M., and Someya, N. (1968). X-ray demonstration of lesions in the anterior wall of the stomach. Stomach and Intestine, 3, 873-882.

Kurokawa, T., Kajitani, T., and Oota, K. (1966). Carcinoma of the Stomach in Early Phase, pp. 11-231. Nakayama-Shoten, Tokyo, Japan.

Shirakabe, H., Kumakura, K., Koyama, R., Nishigawa, M., Horikoshi, H., Higurashi, K., Yamada, T., Doi, H., Sawaguchi, Y., Okubo, H., Yoshida, S., Nakajima, A., and Ichikawa, H. (1961). X-ray diagnosis of gastric and duodenal ulcer. In Proc. Gastroent. Soc., Tokyo.

(1966). Atlas of X-ray Diagnosis of Early Gastric Cancer, 1st ed., lgaku-Shoin, Tokyo, Japan.

Taylor, D. A., Kevin, L., Macken, K. L., Bachman, A. L., and Seaman, W. B., (1965). A new method for visualisation of gastric wall. Radiology, 84, 351-353. 